\title{
Detecting Apoptosis as a Clinical Endpoint for Proof of a Clinical Principle
}

\author{
Piero Zollet ${ }^{a, b}$ Timothy E. Yap ${ }^{a, c}$ M. Francesca Cordeiro ${ }^{a, c, d}$ \\ ${ }^{a}$ The Imperial College Ophthalmology Research Group (ICORG), Imperial College London, London, UK; \\ ${ }^{b}$ Department of Ophthalmology, Università Vita-Salute San Raffaele, Milan, Italy; ${ }^{\mathrm{C}}$ The Western Eye Hospital, \\ Imperial College Healthcare NHS Trust (ICHNT), London, UK; ${ }^{\mathrm{d}}$ Glaucoma and Retinal Degeneration Research Group, \\ Visual Neurosciences, UCL Institute of Ophthalmology, London, UK
}

\section{Keywords}

Apoptosis · Imaging · Retina - Neurodegeneration ·

Glaucoma

\begin{abstract}
The transparent eye media represent a window through which to observe changes occurring in the retina during pathological processes. In contrast to visualising the extent of neurodegenerative damage that has already occurred, imaging an active process such as apoptosis has the potential to report on disease progression and therefore the threat of irreversible functional loss in various eye and brain diseases. Early diagnosis in these conditions is an important unmet clinical need to avoid or delay irreversible sight loss. In this setting, apoptosis detection is a promising strategy with which to diagnose, provide prognosis and monitor therapeutic response. Additionally, monitoring apoptosis in vitro and in vivo has been shown to be valuable for drug development in order to assess the efficacy of novel therapeutic strategies both in the pre-clinical and clinical setting. Detection of Apoptosing Retinal Cells (DARC) technology is to date the only tool of its kind to have been tested in clinical trials, with other new imaging techniques under investigation in the fields of neuroscience, ophthalmology and drug devel-
\end{abstract}

opment. We summarise the transitioning of techniques detecting apoptosis from bench to bedside, along with the future possibilities they encase.

(c) 2020 S. Karger AG, Basel

\section{Clinical Relevance of Apoptosis Detection}

The eye represents a privileged window through which we can view the central nervous system, offering clinicians and researchers the opportunity to use retinal biomarkers in the diagnosis and monitoring of neuronal physiology and pathology in vivo. Apoptosis of retinal cells is the common endpoint of different insults occurring in a variety of neurodegenerative diseases [1]. The archetypal neurodegenerative disease of the retina is glaucoma, characterised by retinal ganglion cell (RGC) apoptosis, although other modes of death have been proposed $[2,3]$. Accompanying RGC loss are retinal nerve fibre layer thinning, optic disc cupping and irreversible loss of visual field which can all be clinically detected [4]. Pathological death of RGCs has also been detected in neurodegenerative conditions such as Alzheimer's disease (AD), Parkinson's disease (PD), optic neuritis and multiple sclerosis. In contrast, other ophthalmic conditions

\footnotetext{
M. Francesca Cordeiro

The Western Eye Hospital Imperial College Healthcare Trust 153-173 Marylebone Road, London NW1 5QH (UK) francesca.cordeiro@nhs.net
} 
may involve different cell populations; for example, in age-related macular degeneration (AMD), retinal pigment epithelium and photoreceptors progressively degenerate, leading to central vision loss [5]. Monitoring the rate of this underlying degenerative process is of great importance in order to guide treatment and indicate prognosis.

RGC loss is a physiological process ubiquitously occurring due to ageing; however, the progression rate of RGC loss is significantly higher in subjects affected by glaucoma [6]. We usually lose approximately $0.4 \%$ of our RGC population per year, while in glaucomatous patients the rate is increased approximately 10 -fold $[6,7]$. On average, a healthy subject has around 1.2 million RGC at birth [6], with approximately $20-40 \%$ thought to be lost before visual field defects are detected [8]. This leads to a diagnostic delay of up to 10 years [9]. Tools to measure the rate of programmed cell death in a minimally invasive manner will hopefully complete the standard eye examination of the future, if they can replace the need for extended follow up that is responsible for the delay in many diagnoses. Moreover, repeated measures of apoptosis detection will hopefully provide an IOP-independent and robust clinical trial biomarker.

\section{Apoptosis within the Glaucoma Paradigm}

The most widely accepted and only currently modifiable factor associated with glaucoma is elevated intraocular pressure (IOP) $[10,11]$. The axons of RGCs gather at the optic disc and cross the sclera at the level of a structure known as the lamina cribrosa. IOP may cause excessive stress on RGC fibres at this level, interrupting the orthograde and retrograde axonal trafficking of nutrients and trophic factors [12]. Moreover, during the periods of elevated IOP, metabolic stress is induced, and energy demands of RGC and astrocytes rise, leading to mitochondrial dysfunction [13]. However, raised IOP is neither necessary nor sufficient in isolation for diagnosis [14]. It is the characteristic RGC death that defines the disease, heralded by apoptosis occurring in order to avoid a destructive localised or systemic inflammatory reaction. Apoptosis-initiating events (such as raised IOP) are followed by cell shrinkage and blebbing, chromatin condensation and DNA fragmentation [15], but a very early event in this process is the translocation of phosphatidylserine (PS) to the external leaflet of the cell membrane [16]; this can be exploited by in vitro and in vivo diagnostic techniques.

Apoptosis Detection in Retinal Diseases

\section{Retinal Cell Apoptosis in Other Eye and Brain Diseases}

A deregulated programmed cell death is thought to occur in other retinal diseases such as AMD [17], diabetic retinopathy (DR), and other retinal dystrophies. In all these conditions, monitoring apoptosis may represent a surrogate biomarker of disease activity and progression. AMD is the leading cause of irreversible blindness in the ageing population [18], and a major worldwide health problem. The primary insult in AMD occurs at the level of RPE, due to accumulation of yellowish autofluorescent lipofuscin deposits above Bruch's membrane and beneath RPE cells, known as drusen. Drusen are responsible for the distortion of central vision in "dry" AMD, the size of which may range from tiny dots up to $250 \mu \mathrm{m}$ [18]. Larger drusen have a tendency to fuse leading to pigment epithelial detachment. This last phenomenon represents a risk factor for the development of the "wet" form of AMD, during which neovascularisation from the choroidal circulation causes exudation and haemorrhage destructive to the anatomical order of the retinal layers. Secondary to RPE degeneration, rod and cone dysfunction also accounts for central vision loss, a characteristic feature of the pathology [18]. Notably, rods are more severely affected by AMD [19], with significant rates of photoreceptor and RPE apoptosis seen with TUNEL staining (terminal deoxynucleotidyl transferase dUTP nick-end labelling) [20].

DR is a common complication of diabetes mellitus. It is the most common cause of vision loss among working age groups and represents a huge socio-economic burden [21]. The most striking pathological changes of DR are the microvascular complications occurring in the retinal tissue; however, DR also involves an increased rate of apoptosis both in vascular and neuro-retinal cells, as shown by TUNEL assay-based studies [22]. The cell populations mostly involved in this phenomenon are RGCs and amacrine cells [23].

The degeneration of some retinal cell populations has been associated with brain disorders such as $\mathrm{AD}$ and $\mathrm{PD}$ [24]. $\mathrm{AD}$ is by far the most common form of dementia, accounting for approximately $70 \%$ of all cases [25]. The main pathological and diagnostic feature is represented by the deposition of extracellular senile plaques and intracellular neurofibrillary tangles. Nowadays, diagnosis is primarily based on the patient's behavioural and clinical assessment [26] and, secondarily, confirmed by either computed tomography or magnetic resonance imaging [27]. The problem of this current approach is related to

Ophthalmologica 2021;244:408-417 409 
the irreversibility of the damage at the time of diagnosis, while by contrast the pathological accumulation of amyloid may have begun 10-15 years earlier [25]. This underlines the need for novel strategies for early disease detection and prevention. The idea of using the eye as a window over the central nervous system for diagnosis of $\mathrm{AD}$ is rapidly growing since retinal nerve fibre layer loss has been widely observed [1]. The detection of amyloid-beta in the retina of $\mathrm{AD}$ patients has produced conflicting results [1]. PD is the second most common cause of dementia worldwide, affecting approximately $1 \%$ of the population over 65 years of age [28]. The histologic hallmark of the disease is the accumulation of protein aggregates, known as Lewy bodies, in the cytoplasm of diseased cells [29]. The most affected cells in the brain are the dopaminergic neurons belonging to the substantia nigra. The degeneration of these cells is responsible for the motor symptoms that are a characteristic feature of the disease [29]. Nevertheless, also other brain areas are involved in this degenerative process, and this accounts for the nonmotor manifestations such as depression, visual problems, sleep disorders, sense of smell alterations and gastrointestinal symptoms [30]. These non-motor symptoms have been shown to arise years earlier than their motor counterpart and may be the prodrome of the fullblown dementia [30]. In AD, PD, Huntington's disease and glaucoma there are common elements such as oxidative stress, mitochondrial dysfunction, excitotoxicity and misfolded protein aggregation. Therefore, there is potential for apoptosis detection as a biomarker of disease diagnosis in all of these diseases [31].

\section{In vivo Apoptosis Detection: Detection of Apoptosing Retinal Cells}

Apoptosis detection in vivo exploits modifications of apoptotic cells undergoing programmed cell death: PS exposure, changes in apoptotic membrane imprint and caspase activation.

Since its first description in 2004, Detection of Apoptosing Retinal Cells (DARC) technology has been used for the assessment and follow-up of different animal models of degenerative retinal diseases for natural history characterisation and the study of novel neuroprotective agents [32]. Moreover, ongoing clinical trials are evaluating DARC performance in human subjects.

The principle of DARC is based on the use of fluorescently labelled annexin A5. Although ubiquitously expressed, annexin A5's function is not fully understood.
However, its ability to bind PS in a calcium-dependent manner is exploited in apoptosis detection [33]. The majority of PS is usually maintained on the intracellular aspect of the cytoplasmic membrane due to the action of ATP-dependent "flippases"; however, increasing translocation to the extracellular surface occurs during cell stress and the process initiating apoptotic cell death, possibly with the involvement of "scramblases" [34]. PS represents an "eat me" signal for phagocytes, removing apoptotic debris to prevent pro-inflammatory consequences [35].

Annexin A5 was first developed for in vitro labelling of apoptosis but was soon re-developed for in vivo imaging of apoptosing tissues using radioactive tracers in combination with positron emission tomography and single-photon emission computed tomography nuclear imaging techniques [36]. The main applications of these types of studies related to oncology, inflammatory bowel diseases, myocardial infarction and strokes [37-39]. For DARC to accomplish retinal imaging, annexin A5 has been fluorescently labelled using both $488-\mathrm{nm}$ and nearinfrared 776-nm tags with excitation and emission spectra of 495-519 and 771-793 nm, respectively (ANX776) [40].

Modified confocal scanning laser ophthalmoscopy (cSLO) is used to image the retina using the in-built fluorescent detection systems, providing high-contrast retinal images [41]. The latter near-infrared wavelength of ANX776 is aligned to that of indocyanine green for which imaging set-ups are in widespread use by medical retina specialists. The field of image acquisition is between 30 and $55^{\circ}$ and can be either centred on the fovea or the optic disc. Compensation for non-enhancing structures and non-linear distortion is performed post-acquisition processing [42], with quantification of apoptosing cells performed via a template-matching approach to count hyperfluorescent spots, a count known as the "DARC count" [43].

\section{DARC Technology in Pre-Clinical and Clinical Studies}

\section{Pre-Clinical Studies Using DARC}

DARC has been used in many pre-clinical studies including animal models of glaucoma and other neurodegenerative conditions to investigate disease pathogenesis and the effectiveness of potential treatments. The first study was published in 2004 demonstrating histological validation and disease activity in a rat model of glaucoma [32]. DARC has also been used to characterise the rela- 
tionship between raised IOP and retinal apoptosis, an initiating injury used in many animal models of glaucoma [44]. Following on from this work, a novel staurosporineinduced rat ocular hypertension model was demonstrated and used to investigate the neuroprotective effect of modulating glutamate-induced excitotoxicity [45]. DARC was used to investigate the role of amyloid plaques in retinal tissue and their relationship with retinal apoptosis. These deposits were related to the rate of apoptosis in a dose- and time-dependent manner, with strategies to prevent amyloid plaque formation or enhancement of their clearance, beneficial in terms of RGC survival [46].

Retinal apoptosis has also been monitored in diabetic mice, which had increased DARC counts in comparison to wild-type controls. These results support the use of in vivo apoptosis detection as an early biomarker of DR, before visible vascular changes are detectable on fundus examination [47].

Photoreceptor loss whilst investigating the role of blue light exposure in dark Agouti rats has been characterised by DARC, revealing hyperfluorescent apoptotic cells in the outer retina, confirmed by histological staining of photoreceptors [48]. This last study prompted further investigation into macular degeneration, where DARC was able to detect the presence of apoptosis in photoreceptors in a mouse model of dry AMD [18].

DARC has been used to investigate the potential of novel therapeutics such as the effectiveness of MRZ99030, a modulator of amyloid-beta aggregation, as a neuroprotector. The study highlighted a dose-dependent reduction of apoptosis upon systemic injection of the molecule [49]. Another study used the partial optic nerve transection (pONT) model to show the ability of 2-Cl-IBMECA, an adenosine A3 agonist, to reduce retinal apoptosis in vivo [50]. Brimonidine, an alpha-2 adrenergic receptor agonist, was shown to be able to reduce the rate of retinal cell apoptosis through an IOP-independent mechanism related to amyloid precursor protein aggregation modulation [51]. Coenzyme Q10 was also shown to facilitate significant reduction of retinal apoptosis in vivo using DARC [52]. A liposomal formulation of rosiglitazone, a peroxisome proliferator-activated receptorgamma agonist, was used with DARC in a rotenone-induced rat model of PD [53]. The results of the study showed protection both at the level of the retina with reduced retinal apoptosis, and in the nigrostriatal pathways of the brain [53]. More recently, topical nanoparticles of memantine, an NMDA receptor antagonist used to treat $\mathrm{AD}$, and curcumin, a naturally occurring polyphenol found in turmeric, have been tested for their neuropro-

Apoptosis Detection in Retinal Diseases tective abilities in vivo [54, 55]. DARC demonstrated both were able to provide significant reduction in retinal apoptosis in rat models of ocular hypertension $[54,55]$. A novel cell-based therapy, the delivery of Schwann cells directly on the damaged optic nerve sheath, was also shown to produce sustained results promoting axon regrowth and preventing secondary RGC neurodegeneration using DARC [56]. Eventually, DARC technology has been used in a recent study to monitor the effects of topical recombinant human nerve growth factor (rh-NGF) in a rat model of glaucoma. The results showed that partial optic nerve transected rats receiving topical rh-NGF had a non-statistically significant difference in RGC apoptosis rate if compared with naive controls, both at DARC count and histologic sample examination [57].

\section{DARC in the Clinical Setting}

\section{Phase I Clinical Trial}

After the promising results in animal models, DARC technology has been tested in the clinical setting, with the phase I clinical trial published in 2017 [40]. This was a single-centre, open-label, proof-of-concept clinical trial designed to primarily assess safety, and secondarily efficacy of DARC imaging in humans [40]. The study was carried out on 8 healthy volunteers and 8 patients affected by progressing glaucoma. These subjects were randomly allocated to one of the different ANX776 dosage groups. Each of the four dosage groups included 2 glaucoma patients and 2 healthy controls. After the single intravenous injection of ANX776, retinal imaging was performed to visualise fluorescently labelled retinal cells at $15,30,60,120,240$, and $360 \mathrm{~min}$ (Fig. 1). Apoptotic retinal cells were identified as hyperfluorescent areas on the retina of a size between 12 and $16 \mu \mathrm{m}$ using a cSLO focused on the RGC layer. DARC spots were objectively counted using a method of template matching to track them longitudinally $[43,58]$.

All subjects were required to attend three visits, and a follow-up at 30 days. They underwent standard eye examination, including best-corrected visual acuity, tonometry, gonioscopy, dilated fundus examination, optical coherence tomography (OCT) and standard automated perimetry. Additionally, all glaucoma patients were regularly followed as part of the standard glaucoma care up to 16 months after DARC. This strategy allowed the investigators to track glaucoma progression and compare the standard indices with DARC, in order to test the potential of DARC as a predictive surrogate marker. 


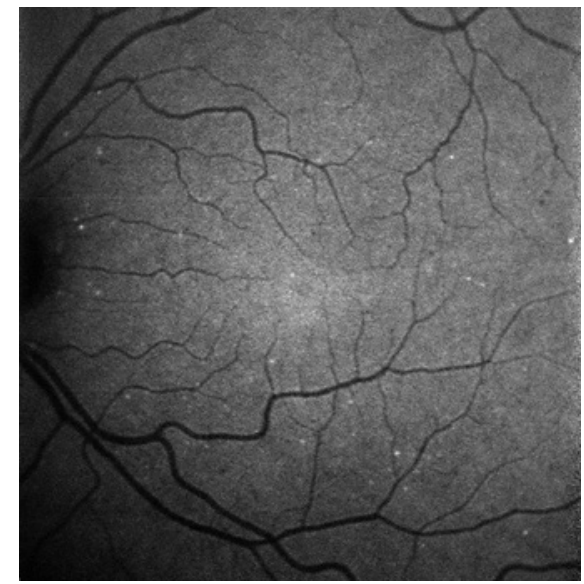

Fig. 1. Detection of hyperfluorescent DARC spots representing retinal apoptosing cells after injection of ANX776. The count of these spots gives an estimation of the rate of RGC degeneration.

No patients were withdrawn from the study, and no serious adverse events occurred. The study reported only isolated cases of discomfort during phlebotomy, hematoma at cannulation site, influenza, metatarsal inflammation and dizziness. ANX776 showed rapid absorption and elimination without accumulation. The half-life of the drug ranged from 18 to $36 \mathrm{~min}$, with maximal concentration proportional to the dose administered. These results were consistent with other studies using radiolabelled annexin A5 [59].

The greatest difference between healthy controls and glaucoma patients was seen when ANX776 was administered at a dose of $0.4 \mathrm{mg}(p<0.01)$. Multivariate analysis showed a 2.4-fold higher DARC count in glaucoma patients across the $6 \mathrm{~h}$ monitored (95\% CI 1.4-4.03; $p=$ $0.003)$. The DARC count was found to be significantly correlated with decreased central corneal thickness, increased cup-disc ratio and increased age. Post hoc it was shown that DARC was able to predict the increased rate of progression, therefore showing the potential prognostic role of this technology.

Overall, this study proves the safety of the intravenous administration of ANX776 in human subjects and suggests the optimal dosage for apoptosis detection. The results suggest DARC technology may have clinical potential for early glaucoma diagnosis, and monitoring for progression and therapeutic success.

\section{Phase II Clinical Trial}

Following the successful results of the phase I clinical trial, DARC imaging technology has undergone phase II clinical evaluation, the results of which are due to be published shortly. It is a single-centre, non-randomised, open-label clinical trial examining the use of ANX766 to image retinal apoptosis in the retinas of healthy volunteers, patients $(n=116)$ affected by glaucoma, AMD, optic neuritis and Down's syndrome (with a pathology similar to $\mathrm{AD}[60])$. The patients enrolled for this study received a single shot of ANX776 at a dose of $0.4 \mathrm{mg}$ and were then imaged at $15 \mathrm{~min}, 2$ and $4 \mathrm{~h}$ after the injection. This primary objective of this study is to assess the DARC count in different pathologies, and further assess DARC's potential in early diagnosis and predictive abilities.

\section{PSVue 550}

PSVue 550 (bis(zinc(II)-dipicolylamine), Zn-DPA) is a synthetic molecule able to bind to PS, conjugated with a fluorophore known as Texas red. The affinity of this probe for PS makes it suitable to transiently visualise apoptosis in the retina, allowing for repeated fluorescent imaging. This molecule has shown efficacy with topical administration in rat and mice models of photoreceptor diseases [61], with no direct retinal toxicity of the probe [61].

In the experiment conducted by Mazzoni et al. [61] eye penetration of PSVue 550 was tested on Royal College of Surgeons (RCS) rats, a well-characterised model of retinal degeneration, and wild-type controls. They were able to show that irrespective of retinal degeneration the dye was able to reach the posterior segment of the eye. However, only apoptotic photoreceptors of RCS rats could be visualised. They tested the penetration of another annexinderived molecule able to tag PS, Polarity Sensitive Indicator of Viability and Apoptosis (pSIVA). It was shown a similar fluorescence pattern upon intravitreal injection of either PSVue 550 or pSIVA; however, only PSVue 550 was able to reach the posterior retina upon topical application. These results were obtained upon histologic sample examination fluorescence microscopy [61].

PSVue 550 toxicity was tested through photoscopic full-field electroretinograms on dark-adapted rats at 3 days after eye drop subministration. Control RCS rats received topical Hanks buffered saline solution. No statistical difference to light response could be detected between the treated and control group [61].

Additionally, live imaging of apoptotic photoreceptors in vivo by whole animal scanning was performed in order to assess whether labelling of apoptotic cells was permanent or transient. The results showed that the peak fluorescence could be detected at $24 \mathrm{~h}$ after topical subministration. Moreover, after $72 \mathrm{~h}$, there was no statistical dif- 
ference in the level of fluorescence of treated and control eyes. Therefore, the transitory nature of PSVue 550 labelling has the potential to be exploited for serial monitoring of retinal degeneration at different time points. Using this imaging technique, they validated the results also obtained in other mice models of degenerative photoreceptor disease such as the MerTK-deficient model and the wild-type rat with a light-induced retinal damage model [61].

To further validate this approach, live imaging of apoptotic photoreceptors through retinal imaging was performed. They were able to show a statistically significant difference in retinal fluorescence in treated versus control eyes [61]. The safety and utility of this approach in human subjects are yet to be reported.

\section{Apoptotic Membrane Imprint Modification}

\section{ApoSense ${ }^{\circledR}$}

Aposense ${ }^{\circledR}$ is a molecular imaging technique using amphipathic low-molecular weight molecules of 300-700 Da. These selectively cross the apoptotic plasma membrane and accumulate in the cytoplasm of dying cells [62]. The hydrophobic region enables the anchoring of these molecules to the lipidic surface of the cell membrane, whilst the hydrophilic region would usually block their entrance into the cytoplasm of non-apoptotic cells. Their accumulation in the cytoplasm has been shown to occur alongside recognised apoptotic events such as PS exposure, caspase activation and the loss of mitochondrial membrane potentials. These compounds can be labelled or rely on intrinsic fluorescence, or undergo labelling with a radioactive moiety. Molecules belonging to this family include N,N'-didansyl-L-cystine, NST-732 and 729, ML-9 and ML-10. The first three contain a dansyl group, while the last two contain an alkyl-malonate molecule [62].

To date, they have mostly been exploited in the preclinical setting. The disease models on which they were tested include $\mathrm{AD}$, amyotrophic lateral sclerosis [63], melanomas [64], chemotherapy-induced enteropathy [65] and reperfusion-induced damage models [66]. In the clinical setting, a radiolabelled version of ML-10 has been used to monitor the response to radiotherapy of brain metastases [67].

These molecules are able to cross the blood-brain barrier, and are therefore theoretically suitable for use in neurodegenerative conditions such as $\mathrm{AD}, \mathrm{PD}$, and glaucoma; however, they have never been tested on humans for this purpose. Moreover, a high dose is required to reach desirable image performance in most cases, therefore raising concern regarding the possible toxic events related to their use.

\section{Caspase Activation Detection}

\section{FLIVO $^{T M}$}

Caspases are a family of endoproteases that have a fundamental role in apoptotic and inflammatory processes. In apoptosis, caspase cascade activation occurs through extrinsic or intrinsic signals. The extrinsic pathway is triggered by ligands binding to extracellular death receptors, whilst the intrinsic pathway responds to intracellular stress signals such as hypoxia, DNA damage, reactive oxygen species, misfolded protein accumulation and mitochondrial damage. Irrespective of the trigger, the cascade begins with activation of "initiator" caspases that, once active, are able to cleave and switch on "executioner" caspases. Executioner caspases are responsible for DNA fragmentation which eventually leads to cell death.

FLIVO (fluorescence in vivo) is a family of fluorescent caspase inhibitors that allow visualisation of in vivo and in vitro apoptosis. These tracers can be directly injected into the circulation and selectively accumulate in apoptotic cells. Being able to also cross the blood-brain barrier, they have potential in the study of brain and ocular neurodegenerative conditions by selectively highlighting cells undergoing caspase-dependent apoptosis, the majority of programmed cell death [68].

FLIVO technology has been used only in the pre-clinical setting for in vitro and in vivo studies. It has been exploited in oncology to develop new chemotherapeutic agents and cancer vaccines. In ophthalmology, FLIVO has been used to monitor the activity of DR [69], glaucoma $[70,71]$, retinitis pigmentosa [72], blue-light induced retinal damage and AMD [73].

\section{Z-DEVD-Aminoluciferin}

Luciferins are a family of bioluminescent molecules found naturally in animals such as fireflies. Luciferins are activated via enzymatic cleavage by luciferases. Activated luciferins are able to release energy through light emission. Z-DEVD-aminoluciferin is a modified luciferin. As a caspase $3 / 7$ substrate, it is not activated by luciferases but rather by these specific caspases, and therefore has been used to monitor the activity of these caspases in vitro [74]. Upon cleavage by the aforementioned enzymes, the subsequent bioluminescence is exploited as a marker of 


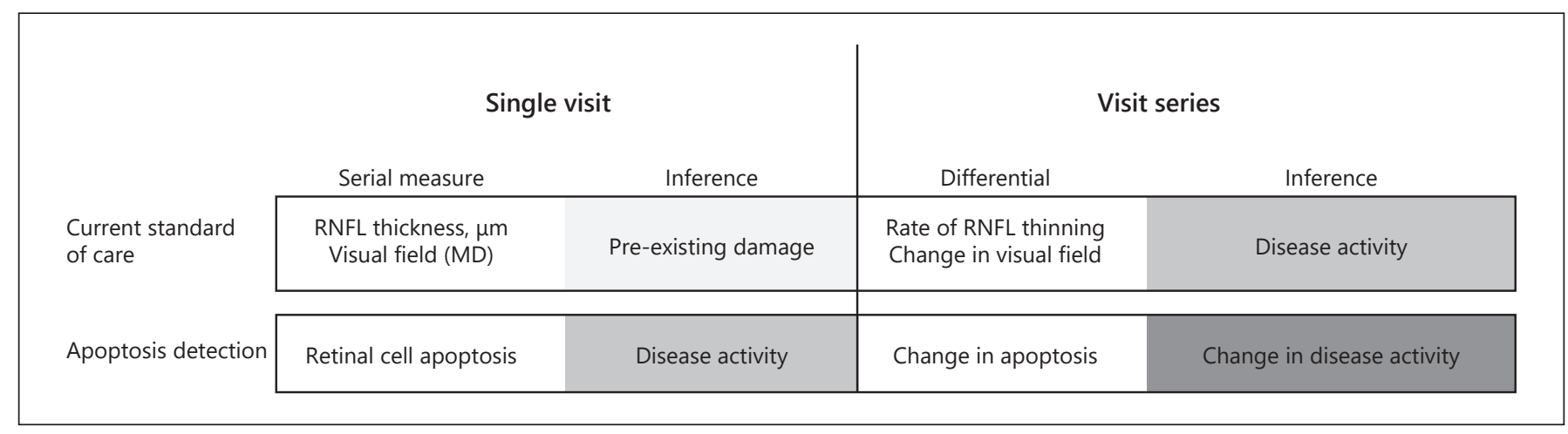

Fig. 2. Demonstration of how clinical apoptosis detection could save time in the diagnostic process by providing an instantaneous measure of disease activity. An additional dimension is added to the diagnostic panel: the rate of change of cell numbers (change in disease activity) can be studied, for example in response to a therapeutic intervention.

apoptosis. In vivo apoptosis detection with an injection of Z-DEVD-aminoluciferin has been shown in mice models of tumour xenografts [75]. This tool has also been used in the drug development setting; however, its role in the clinical field has not been explored yet.

\section{Diagnosis}

The current clinical gold-standard for glaucoma diagnosis is standard automated perimetry. However, this method possesses practical and analytical disadvantages in that it requires a long time to conduct, requires serial measures over several years to detect change, is subject to a learning process that is variable according to the patient's fitness, concentration and comprehension $[76,77]$. Moreover, some SITA protocols, such as the 24-2, have been advocated as inadequate for early disease diagnosis [78]. In the time it takes for a patient to develop a visual field defect detectable by current methods, approximately $30 \%$ of RGCs are lost. The timespan of this pre-clinical phase has been estimated to range from 2 to 8 years, according to the progression rate [6]. In this setting, structural OCT imaging has been shown to be the most promising detector of pre-perimetric RGC loss. This is not only in glaucoma, but also in other neurodegenerative conditions such as AD, PD and optic neuritis [1]. However, the considerable variation between individuals limits the diagnostic value of single measures [79, 80].

Raised IOP has been shown to be associated with progression of visual field defects $[10,80]$. Therefore, the majority of medical and surgical treatments for glaucoma target IOP, with the aim of reducing it; however, it is an imperfect surrogate due to the wide interindividual variability of its pathologic effects [81].
In contrast to the biomarkers discussed above, DARC has the potential to minimise the number of years required to make a diagnosis by offering an indication of the severity of disease on first visit, potentially prior to the formation of significant visual field defects. The imaging of an active process implies prognostic value, and is arguably an important drawback of OCT and perimetry, whereby any defects seen are not necessarily progressive (Fig. 2). It is still unknown if prognosis can also be determined in other neurodegenerative conditions. Additionally, DARC is practical to implement, and not so reliant on the patient's ability to conduct the test. The inter-individual variability and change in disease states that will determine diagnostic ability, and the repeatability of the test that will determine the potential in clinical trials are to be determined in future studies and publications.

\section{Conclusion}

Several ocular and extraocular neurodegenerative diseases share the common feature of early and pathological death of retinal cells. This provides a potential early diagnosis window in which to delay and possibly halt pathologic processes before they cause significant harm. Apoptosis detection in retinal cells seems a plausible means to achieve this goal, with different strategies and technologies in the pipeline, with DARC already proven safe in humans. Their transition from bench to bedside may in the near future aid diagnosis, prognosis, follow-up, therapeutic tailoring and drug development in the field of ophthalmology and neurology. 


\section{Conflict of Interest Statement}

M.F.C. is a named co-inventor on granted patent EP 2231199B1 and published patent WO $2011055121 \mathrm{~A} 1$ owned by UCL and related to DARC technology. The other authors declare no conflicts of interests.

\section{Funding Sources}

No funding was provided for this work.

\section{Author Contributions}

P.Z., writing of the manuscript. T.E.Y., writing and review of the manuscript and the figures. M.F.C., review of the manuscript.

\section{References}

1 Yap TE, Balendra SI, Almonte MT, Cordeiro MF. Retinal correlates of neurological disorders. Ther Adv Chronic Dis. 2019 Dec;10: 2040622319882205.

2 Bergsbaken T, Fink SL, Cookson BT. Pyroptosis: host cell death and inflammation. Nat Rev Microbiol. 2009 Feb;7(2):99-109.

3 Peng JJ, Song WT, Yao F, Zhang X, Peng J, Luo XJ, et al. Involvement of regulated necrosis in blinding diseases: focus on necroptosis and ferroptosis. Exp Eye Res. 2020 Feb;191: 107922.

4 Weinreb RN, Aung T, Medeiros FA. The pathophysiology and treatment of glaucoma: a review. JAMA. 2014 May;311(18):1901-11.

5 Dunaief JL, Dentchev T, Ying GS, Milam AH. The role of apoptosis in age-related macular degeneration. Arch Ophthalmol. 2002 Nov; 120(11):1435-42.

6 Medeiros FA, Lisboa R, Weinreb RN, Liebmann JM, Girkin C, Zangwill LM. Retinal ganglion cell count estimates associated with early development of visual field defects in glaucoma. Ophthalmology. 2013 Apr;120(4): 736-44.

7 Hirooka K, Izumibata S, Ukegawa K, Nitta E, Tsujikawa A. Estimating the rate of retinal ganglion cell loss to detect glaucoma progression: an observational cohort study. Medicine. 2016;95(30):e4209.

8 Kerrigan-Baumrind LA, Quigley HA, Pease ME, Kerrigan DF, Mitchell RS. Number of ganglion cells in glaucoma eyes compared with threshold visual field tests in the same persons. Invest Ophthalmol Vis Sci. 2000 Mar;41(3):741-8.

9 Ahmad SS. An introduction to DARC technology. Saudi J Ophthalmol. 2017 Jan-Mar; 31(1):38-41.

10 Kass MA, Heuer DK, Higginbotham EJ, Johnson CA, Keltner JL, Miller JP, et al. The Ocular Hypertension Treatment Study: a randomized trial determines that topical ocular hypotensive medication delays or prevents the onset of primary open-angle glaucoma. Arch Ophthalmol. 2002 Jun;120(6):701-13.

11 Heijl A, Leske MC, Bengtsson B, Hyman L, Bengtsson B, Hussein M; Early Manifest Glaucoma Trial Group. Reduction of intraocular pressure and glaucoma progression: results from the Early Manifest Glaucoma Trial. Arch Ophthalmol. 2002 Oct;120(10): 1268-79.
12 Fechtner RD, Weinreb RN. Mechanisms of optic nerve damage in primary open angle glaucoma. Surv Ophthalmol. 1994 Jul-Aug; 39(1):23-42.

13 Ju WK, Kim KY, Lindsey JD, Angert M, Duong-Polk KX, Scott RT, et al. Intraocular pressure elevation induces mitochondrial fission and triggers OPA1 release in glaucomatous optic nerve. Invest Ophthalmol Vis Sci. 2008 Nov;49(11):4903-11.

14 Collaborative Normal-Tension Glaucoma Study Group. Comparison of glaucomatous progression between untreated patients with normal-tension glaucoma and patients with therapeutically reduced intraocular pressures [published correction appears in Am J Ophthalmol. 1999 Jan;127(1):120]. Am J Ophthalmol. 1998;126(4):487-97. doi: 10.1016/s00029394(98)00223-2

15 Fang EF, Scheibye-Knudsen M, Chua KF, Mattson MP, Croteau DL, Bohr VA. Nuclear DNA damage signalling to mitochondria in ageing. Nat Rev Mol Cell Biol. 2016 May; 17(5):308-21.

16 Lebois M, Josefsson EC. Regulation of platelet lifespan by apoptosis. Platelets. 2016 Sep; 27(6):497-504.

17 Thomas CN, Berry M, Logan A, Blanch RJ, Ahmed Z. Caspases in retinal ganglion cell death and axon regeneration. Cell Death Discov. 2017 Jul;3(1):17032.

18 Normando EM, Tilley M, Guo L, Cordeiro MF. Imaging in DRY AMD. Drug Discov Today Ther Strateg. 2013;10(1):e35-41.

19 Mitchell P, Liew G, Gopinath B, Wong TY. Age-related macular degeneration. Lancet. 2018 Sep;392(10153):1147-59.

20 Elizabeth Rakoczy P, Yu MJ, Nusinowitz S, Chang B, Heckenlively JR. Mouse models of age-related macular degeneration. Exp Eye Res. 2006 May;82(5):741-52.

21 Lee R, Wong TY, Sabanayagam C. Epidemiology of diabetic retinopathy, diabetic macular edema and related vision loss. Eye Vis (Lond). 2015 Sep;2(1):17.

22 Mizutani M, Kern TS, Lorenzi M. Accelerated death of retinal microvascular cells in human and experimental diabetic retinopathy. J Clin Invest. 1996 Jun;97(12):2883-90.

23 Kern TS, Barber AJ. Retinal ganglion cells in diabetes. J Physiol. 2008;586(18):4401-8.
24 MacCormick IJ, Czanner G, Faragher B. Developing retinal biomarkers of neurological disease: an analytical perspective. Biomarkers Med. 2015;9(7):691-701.

25 Holtzman DM, Morris JC, Goate AM. Alzheimer's disease: the challenge of the second century. Sci Transl Med. 2011 Apr;3(77): 77sr1. https://doi.org/10.1126/scitranslmed. 3002369 .

26 McKhann GM, Knopman DS, Chertkow H, Hyman BT, Jack CR Jr, Kawas CH, et al. The diagnosis of dementia due to Alzheimer's disease: recommendations from the National Institute on Aging-Alzheimer's Association workgroups on diagnostic guidelines for $\mathrm{Al}$ zheimer's disease. Alzheimers Dement. 2011 May;7(3):263-9.

27 Johnson KA, Fox NC, Sperling RA, Klunk WE. Brain imaging in Alzheimer disease. Cold Spring Harb Perspect Med. 2012 Apr; 2(4):a006213

28 Wirdefeldt K, Adami HO, Cole P, Trichopoulos D, Mandel J. Epidemiology and etiology of Parkinson's disease: a review of the evidence. Eur J Epidemiol. 2011 Jun;26(S1 Suppl 1):S158.

29 Braak H, Ghebremedhin E, Rüb U, Bratzke H, Del Tredici K. Stages in the development of Parkinson's disease-related pathology. Cell Tissue Res. 2004 Oct;318(1):121-34.

30 Mahlknecht P, Seppi K, Poewe W. The concept of prodromal Parkinson's disease. J Parkinsons Dis. 2015;5(4):681-97.

31 Cordeiro MF, Guo L, Coxon KM, Duggan J, Nizari S, Normando EM, et al. Imaging multiple phases of neurodegeneration: a novel approach to assessing cell death in vivo. Cell Death Dis. 2010;1(1):e3.

32 Cordeiro MF, Guo L, Luong V, Harding G, Wang $\mathrm{W}$, Jones HE, et al. Real-time imaging of single nerve cell apoptosis in retinal neurodegeneration. Proc Natl Acad Sci USA. 2004 Sep;101(36):13352-6.

33 Meers P, Mealy T. Calcium-dependent annexin $V$ binding to phospholipids: stoichiometry, specificity, and the role of negative charge. Biochemistry. 1993 Nov;32(43): 11711-21.

34 Hankins HM, Baldridge RD, Xu P, Graham TR. Role of flippases, scramblases and transfer proteins in phosphatidylserine subcellular distribution. Traffic. 2015;16(1):35-47. 
35 Fadok VA, Voelker DR, Campbell PA, Cohen JJ, Bratton DL, Henson PM. Exposure of phosphatidylserine on the surface of apoptotic lymphocytes triggers specific recognition and removal by macrophages. J Immunol. 1992 Apr;148(7):2207-16.

36 Petrovsky A, Schellenberger E, Josephson L, Weissleder R, Bogdanov A Jr. Near-infrared fluorescent imaging of tumor apoptosis. Cancer Res. 2003 Apr;63(8):1936-42.

37 Van den Brande JM, Koehler TC, Zelinkova $\mathrm{Z}$, Bennink RJ, te Velde AA, ten Cate FJ, et al. Prediction of antitumour necrosis factor clinical efficacy by real-time visualisation of apoptosis in patients with Crohn's disease. Gut. 2007 Apr;56(4):509-17.

38 Belhocine T, Steinmetz N, Hustinx R, Bartsch $P$, Jerusalem G, Seidel L, et al. Increased uptake of the apoptosis-imaging agent $(99 \mathrm{~m}) \mathrm{Tc}$ recombinant human Annexin V in human tumors after one course of chemotherapy as a predictor of tumor response and patient prognosis. Clin Cancer Res. 2002 Sep;8(9): 2766-74.

39 Hofstra L, Liem IH, Dumont EA, Boersma $\mathrm{HH}$, van Heerde WL, Doevendans PA, et al. Visualisation of cell death in vivo in patients with acute myocardial infarction. Lancet. 2000 Jul;356(9225):209-12.

40 Cordeiro MF, Normando EM, Cardoso MJ, Miodragovic S, Jeylani S, Davis BM, et al. Real-time imaging of single neuronal cell apoptosis in patients with glaucoma. Brain. 2017 Jun;140(6):1757-67.

41 Sahu C. Confocal scanning laser ophthalmoscope. In: Comprehensive notes in ophthalmology. New Delhi: Jaypee; 2011.

42 Yap TE, Donna P, Almonte MT, Cordeiro MF. Real-Time Imaging of Retinal Ganglion Cell Apoptosis. Cells. 2018 Jun;7(6):E60.

43 Brunelli R. Template matching techniques in computer vision: theory and practice. Chichester: Wiley; 2009.

44 Guo L, Moss SE, Alexander RA, Ali RR, Fitzke FW, Cordeiro MF. Retinal ganglion cell apoptosis in glaucoma is related to intraocular pressure and IOP-induced effects on extracellular matrix. Invest Ophthalmol Vis Sci. 2005 Jan;46(1):175-82.

45 Guo L, Salt TE, Maass A, Luong V, Moss SE, Fitzke FW, et al. Assessment of neuroprotective effects of glutamate modulation on glaucoma-related retinal ganglion cell apoptosis in vivo. Invest Ophthalmol Vis Sci. 2006 Feb; 47(2):626-33.

46 Guo L, Salt TE, Luong V, Wood N, Cheung $\mathrm{W}$, Maass $\mathrm{A}$, et al. Targeting amyloid- $\beta$ in glaucoma treatment. Proc Natl Acad Sci USA. 2007 Aug; 104(33):13444-9.

47 Borrie SC, Cheung W, Guo L, Barber AJ, Singh RS, Gardner TW, et al. Diabetic retinal neurodegeneration: in vivo imaging of retinal ganglion cell apoptosis in the Ins2Akita/J mouse. Invest Ophthalmol Vis Sci. 2008;49: 4924.
48 Schmitz-Valckenberg S, Guo L, Cheung W, Moss SE, Fitzke FW, Cordeiro MF. In vivo imaging of retinal cell apoptosis following acute light exposure. Ophthalmologe. 2010 Jan;107(1):22-9. German.

49 Salt TE, Nizari S, Cordeiro MF, Russ H, Danysz W. Effect of the A $\beta$ aggregation modulator MRZ-99030 on retinal damage in an animal model of glaucoma. Neurotox Res. 2014 Nov;26(4):440-6.

50 Galvao J, Elvas F, Martins T, Cordeiro MF, Ambrósio AF, Santiago AR. Adenosine A3 receptor activation is neuroprotective against retinal neurodegeneration. Exp Eye Res. 2015 Nov; 140:65-74.

51 Nizari S, Guo L, Davis BM, Normando EM, Galvao J, Turner LA, et al. Non-amyloidogenic effects of $\alpha 2$ adrenergic agonists: implications for brimonidine-mediated neuroprotection. Cell Death Dis. 2016 Dec;7(12):e2514.

52 Davis BM, Tian K, Pahlitzsch M, Brenton J, Ravindran N, Butt G, et al. Topical coenzyme Q10 demonstrates mitochondrial-mediated neuroprotection in a rodent model of ocular hypertension. Mitochondrion. 2017 Sep;36: $114-23$.

53 Normando EM, Davis BM, De Groef L, Nizari $S$, Turner LA, Ravindran N, et al. The retina as an early biomarker of neurodegeneration in a rotenone-induced model of Parkinson's disease: evidence for a neuroprotective effect of rosiglitazone in the eye and brain. Acta Neuropathol Commun. 2016 Aug;4(1):86.

54 Sánchez-López E, Egea MA, Davis BM, Guo L, Espina M, Silva AM, et al. MemantineLoaded PEGylated Biodegradable Nanoparticles for the Treatment of Glaucoma. Small. 2018 Jan; 14(2): 1701808.

55 Davis BM, Pahlitzsch M, Guo L, Balendra S, Shah P, Ravindran N, et al. Topical Curcumin Nanocarriers are Neuroprotective in Eye Disease. Sci Rep. 2018 Jul;8(1):11066.

56 Guo L, Davis B, Nizari S, Normando EM, Shi $\mathrm{H}$, Galvao J, et al. Direct optic nerve sheath (DONS) application of Schwann cells prolongs retinal ganglion cell survival in vivo. Cell Death Dis. 2014 Oct;5(10):e1460.

57 Guo L, Davis BM, Ravindran N, Galvao J, Kapoor N, Haamedi N, et al. Topical recombinant human Nerve growth factor (rh-NGF) is neuroprotective to retinal ganglion cells by targeting secondary degeneration. Sci Rep. $2020 \mathrm{Feb} ; 10(1): 3375$.

58 Adanja I, Debeir O, Mégalizzi V, Kiss R, Warzée N, Decaestecker C. Automated tracking of unmarked cells migrating in three-dimensional matrices applied to anti-cancer drug screening. Exp Cell Res. 2010 Jan;316(2):18193.

59 Benali K, Louedec L, Azzouna RB, Merceron $\mathrm{O}$, Nassar P, Al Shoukr F, et al. Preclinical validation of 99mTc-annexin A5-128 in experimental autoimmune myocarditis and infective endocarditis: comparison with $99 \mathrm{mTc}$ HYNIC-annexin A5. Mol Imaging. 2014;13.
60 Head E, Lott IT, Wilcock DM, Lemere CA. Aging in Down syndrome and the development of Alzheimer's disease neuropathology. Curr Alzheimer Res. 2016;13(1):18-29.

61 Mazzoni F, Müller C, DeAssis J, Lew D, Leevy WM, Finnemann SC. Non-invasive in vivo fluorescence imaging of apoptotic retinal photoreceptors. Sci Rep. 2019 Feb;9(1):1590.

62 Grimberg H, Levin G, Shirvan A, Cohen A, Yogev-Falach M, Reshef A, et al. Monitoring of tumor response to chemotherapy in vivo by a novel small-molecule detector of apoptosis. Apoptosis. 2009 Mar;14(3):257-67.

63 Shirvan A, Reshef A, Yogev-Falach M, Ziv I. Molecular imaging of neurodegeneration by a novel cross-disease biomarker. Exp Neurol. 2009 Sep;219(1):274-83.

64 Cohen A, Ziv I, Aloya T, Levin G, Kidron D, Grimberg $\mathrm{H}$, et al. Monitoring of chemotherapy-induced cell death in melanoma tumors by N,N'-Didansyl-L-cystine. Technol Cancer Res Treat. 2007 Jun;6(3):221-34.

65 Levin G, Shirvan A, Grimberg H, Reshef A Yogev-Falach M, Cohen A, et al. Novel fluorescence molecular imaging of chemotherapy-induced intestinal apoptosis. J Biomed Opt. 2009 Sep-Oct;14(5):054019.

66 Damianovich M, Ziv I, Heyman SN, Rosen S, Shina A, Kidron D, et al. ApoSense: a novel technology for functional molecular imaging of cell death in models of acute renal tubular necrosis. Eur J Nucl Med Mol Imaging. 2006 Mar;33(3):281-91.

67 Höglund J, Shirvan A, Antoni G, Gustavsson SÅ, Långström B, Ringheim A, et al. 18F-ML10, a PET tracer for apoptosis: first human study. J Nucl Med. 2011 May;52(5):720-5.

68 Griffin RJ, Williams BW, Bischof JC, Olin M, Johnson GL, Lee BW. Use of a fluorescently labeled poly-caspase inhibitor for in vivo detection of apoptosis related to vascular-targeting agent arsenic trioxide for cancer therapy. Technol Cancer Res Treat. 2007 Dec;6(6): 651-4.

69 Perrone L, Devi TS, Hosoya KI, Terasaki T, Singh LP. Inhibition of TXNIP expression in vivo blocks early pathologies of diabetic retinopathy. Cell Death Dis. 2010 Aug;1(8):e65.

70 Marshall J, Wong KY, Rupasinghe CN, Tiwari $\mathrm{R}$, Zhao X, Berberoglu ED, et al. Inhibition of $\mathrm{N}$-Methyl-D-aspartate-induced retinal neuronal death by polyarginine peptides is linked to the attenuation of stress-induced hyperpolarization of the inner mitochondrial membrane potential. J Biol Chem. 2015 Sep; 290(36):22030-48.

71 Bosco A, Crish SD, Steele MR, Romero CO, Inman DM, Horner PJ, et al. Early reduction of microglia activation by irradiation in a model of chronic glaucoma. PLoS One. 2012; 7(8):e43602.

72 Subramani M, Murugeswari P, Dhamodaran K, Chevour P, Gunasekaran S, Kumar RS, et al. Short pulse of clinical concentration of bevacizumab modulates human retinal pigment epithelial functionality. Invest Ophthalmol Vis Sci. 2016 Mar;57(3):1140-52. 
73 Dib B, Lin H, Maidana DE, Tian B, Miller JB, Bouzika $\mathrm{P}$, et al. Mitochondrial DNA has a pro-inflammatory role in AMD. Biochim Biophys Acta. 2015 Nov; 1853(11 11 Pt A): 2897-906.

74 O'Brien MA, Daily WJ, Hesselberth PE, Moravec RA, Scurria MA, Klaubert DH, et al. Homogeneous, bioluminescent protease assays: caspase-3 as a model. J Biomol Screen. 2005 Mar; 10(2):137-48.

75 Scabini M, Stellari F, Cappella P, Rizzitano S, Texido G, Pesenti E. In vivo imaging of early stage apoptosis by measuring real-time caspase-3/7 activation. Apoptosis. 2011 Feb; 16(2):198-207.
76 Gardiner SK, Demirel S, Johnson CA. Is there evidence for continued learning over multiple years in perimetry? Optom Vis Sci. 2008 Nov; 85(11):1043-8

77 Birt CM, Shin DH, Samudrala V, Hughes BA, Kim C, Lee D. Analysis of reliability indices from Humphrey visual field tests in an urban glaucoma population. Ophthalmology. 1997 Jul;104(7):1126-30.

78 De Moraes CG, Hood DC, Thenappan A, Girkin CA, Medeiros FA, Weinreb RN, et al. 24-2 visual fields miss central defects shown on 10-2 tests in glaucoma suspects, ocular hypertensives, and early glaucoma. Ophthalmology. 2017;124(10):1449-56.
79 Kuang TM, Zhang C, Zangwill LM, Weinreb RN, Medeiros FA. Estimating lead time gained by optical coherence tomography in detecting glaucoma before development of visual field defects. Ophthalmology. 2015 Oct; 122(10):2002-9.

80 Yu M, Lin C, Weinreb RN, Lai G, Chiu V, Leung CK. Risk of Visual Field Progression in Glaucoma Patients with Progressive Retinal Nerve Fiber Layer Thinning: A 5-Year Prospective Study. Ophthalmology. 2016 Jun; 123(6):1201-10.

81 Anderson DR, Drance SM, Schulzer M; Collaborative Normal-Tension Glaucoma Study Group. The effectiveness of intraocular pressure reduction in the treatment of normaltension glaucoma. Am J Ophthalmol. 1998 Oct;126(4):498-505. 